\title{
Factors influencing clinician's antibiotic prescribing behaviors (apb) in Bangladesh: an in-depth review using comb model
}

\begin{abstract}
This review aimed to summarize determinants influencing clinician's antibiotic prescribing behaviors in Bangladesh. The 18 published articles and grey literature were selected from scientific databases of Web of Science, Global Health, Medline, Pub Med, the Lancet, Cochrane Library and websites of some health organizations and Bangladesh Ministry of Health. A conceptual framework COMB model was utilized to identify and analyze determinants influencing antibiotics prescribing behaviors. The findings of the review revealed that there is a considerable gap in individual-level knowledge, skills, and awareness to rational antibiotic prescribing among physicians. Lack of training, no provision of prescription auditing, and patient load are key barriers to standard antibiotic prescribing practices. The patient's ignorance and expectation of antibiotics, antibiotics promotion with misleading information and financial incentives by pharmaceutical companies potentially motivate prescribers' decision to prescribe even expensive and unnecessary antibiotics. Moreover, lack of adoption and implementation of regulatory policies intensify poor antibiotic prescribing attitude in Bangladesh. Determinants to APB are multiple, multileveled and multi-complexes. There is no alternative without developing pragmatic interventions (educational and regulatory) targeted to key barriers improving APB at healthcare level in Bangladesh. It is recommended to key stakeholders, academia, and key implementers; DGHS, DGDA, and MOHFW of the country in adopting a national action plan to control imprudent antibiotic prescribing behaviors by clinicians and implementing interventions from local to national level at healthcare institutions with an urgent policy action - key to tackle antibiotic resistance in future.
\end{abstract}

Keywords: antibiotics, prescribing behavior, determinants, bangladesh, COMB model
Volume I Issue 4 - 2017

\author{
Sajal Kumar Saha, ${ }^{1,2}$ Shukla Promite ${ }^{3}$ \\ 'Leeds Institute of Health Science, University of Leeds, United \\ Kingdom \\ ${ }^{2}$ Department of Clinical Pharmacy and Pharmacology, University \\ of Dhaka, Bangladesh \\ ${ }^{3}$ Department of Microbiology, University of Dhaka, Bangladesh
}

Correspondence: Sajal Kumar Saha, Department of Clinical Pharmacy and Pharmacology, University of Dhaka, Dhaka, Bangladesh, Tel +610452639559, Email sajal.saha@monash.edu

Received: September 29, 2017 | Published: December 06, 2017
Abbreviations: APB, antibiotics prescribing behavior; AR, antibiotic resistance; AUB, antibiotic use behavior; $\mathrm{CDC}$, centre for disease control; DGHS, directorate general health services; DGDA, directorate general of drug administration; MOHFW, ministry of health and family welfare; NICE, national institute for clinical excellence

\section{Introduction}

Over the last decade, there are poly-pharmacy practices and extensive uses of antibiotics have become a commonplace in Bangladesh. ${ }^{1}$ Overprescribing and unnecessary prescribing of antibiotics is a threatening problem in Bangladesh and worldwide. ${ }^{2}$ The inappropriate use of antibiotics and development of antibiotic resistance (AR) are closely linked. ${ }^{3,4}$ The annual death toll for AR is 700,000 globally. ${ }^{5}$ Nowadays, AR is, therefore, a current global threat to public health. Therefore, promoting appropriate use of antibiotics is an emerging issue for patient safety and public health with national priority. ${ }^{6}$ In 2014, Lundborg \& Tamhanker ${ }^{7,8}$ stated that human behavior is vigorously involved using antibiotics and developing antibiotic resistance. There are many decisions, which are taken prior to antibiotic prescription, dispensing, consumption or discarding influence subsequent human behavior. Individual involved might be a physician, health care provider, a veterinarian, an animal provider, a consumer, or a parent. The engagement of a human behavior is also observed in the non-human use of antibiotics in animal husbandry, industries, and agriculture.

However, it is a neglected area of attention despite having a very important part of antibiotic consumption. ${ }^{8}$ Additionally, antibiotic research and development, manufacturing and sales, which influence antibiotics, use behavior (AUB) of humans. The misuse, abuse, and overuse of antibiotics provide selective pressure on developing enormous antibiotic-resistant bacteria causing environmental pollution. ${ }^{9}$ Bangladesh is no exception. Thus, infectious disease control is under threat due to AR in Bangladesh. ${ }^{2}$ Therefore, health promotion approach is inevitably today's actionable step in Bangladesh to change human (prescribers and users) AUB as a measure of containment of antibiotic resistance. However, to adopt innovative and effective interventions at the line of health promotion, it is essential to uncover key determinants to inappropriate APB in Bangladesh. Hence, an indepth review has been done with the aim of summarizing concrete factors behind clinicians' irrational APB in health care practices in Bangladesh based on yet studied literature.

\section{Discussion}

\section{Methodology}

Literature search strategy: The search strategy was designed to identify relevant studies addressing AR; reasons of over-prescribing 
and factors influencing inappropriate prescribing of antibiotics. The keywords and their equivalents with truncation used in individual databases. To obtain relevant references, it was accomplished a computerized search through scientific databases of Web of Science, Global Health, MEDLINE-Pub MED and The Cochrane Library from January 1982 to December 2016. In addition, it was manually searched in Google for reports, recommendations or editorials posted on official websites of World Health Organization (WHO), Centre for Disease Control and Prevention (CDC), Public Health England (PHE), Ministry of Health, Bangladesh and Directorate General of Drug Administration (DGDA) websites.

Determinant analysis methods: The very complex process of antibiotics use is influenced by ample of factors which affect all engaged role players of physicians, other healthcare providers, healthcare system, patients and the general public and again such determinants are mutually dependent. ${ }^{10}$ It was considered a COM-B framework $^{11}$ to analyze determinants to explain APB from a broader perspective. This review considered 18 literatures to analyze factors influencing APB in Bangladesh (Figure 1).

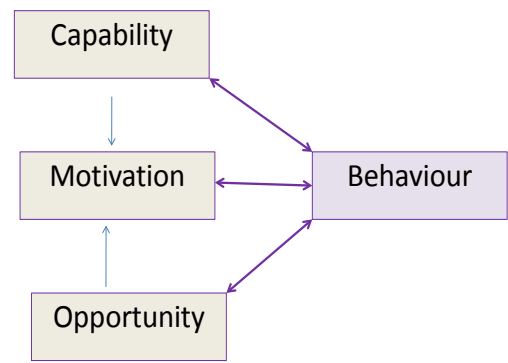

Figure I COMB framework to explain APB. ${ }^{10}$

COMB framework: Capability, motivation, and opportunity are called "components" which collectively influence behaviors (Figure 1). Capability represents 'individual's psychological and physical capacity to engage in the activity concerned. ${ }^{11}$ Capability components have been indicated prescribers' psychological and physical capability to diagnose diseases or complications correctly in such a way so that they can decide where antibiotics are really required and what type and doses of antibiotics are of interest to optimize outcomes. Opportunity covers determinants that stand outside the individual that prompt or make possible the behavior. Thus, it includes aspects of individuals (prescribers') physical (provided by the environment) and social opportunities (cultural milieu that dictates the way we think about things) that may influence antibiotic prescribing behaviors (APB). Motivation comprises 'all those brain processes that energize and direct behavior, not just goals and conscious decision-making' but also 'habitual processes, emotional responding' and 'analytical decision-making. ${ }^{11}$ Motivation is subdivided into reflective motivation (evaluations and plans) and automatic motivation (emotions and impulses arising from associative learning and or innate dispositions) which may play roles to the prescribers antibiotic prescribing decisions.

\section{Results}

After database search and full text review, 18 articles were included for data extraction and analysis using COMB model. The four studies ${ }^{1,12-14}$ reported that there is a lack of enough knowledge and skills to identify the situations of patients where antibiotics are necessary. Even some studies stated that prescribers have lack of awareness against antimicrobial resistance. Five studies reported that there is a considerable shortage of physical facilities like antibiotic guidelines, CPD training, updated antibiotic resistance data in place of practice. ${ }^{15-18}$

\section{Primary analysis}

Physical capability (CPh): In Bangladesh, indiscriminate and inappropriate prescription of antibiotics against common cold and fever of viral origin is due to lack of skills of the registered prescribers. ${ }^{2}$ Ahmed et al. ${ }^{1}$ state that the major health care providers of rural areas are informal sector practitioners in Bangladesh; 43\% traditional healers, 7\% Community Health Workers (CHWs) and $16 \%$ are unqualified allopathic providers (village doctors and drug sellers). Their poor or no professional knowledge on common illnesses influence misuse, abuse and overuse behavior of antibiotics. For instance, trained CHWs by the NGOs (46\%) are comparatively better in the rational use of antibiotics than the unqualified allopathic providers. ${ }^{12}$

Psychological capability (CPs): Knowledge and attitude of the prescribers are key determinants of the ability to prescribe antibiotics rationally. ${ }^{13}$ Physicians' knowledge of quality prescribing of antibiotics depends on previous clinical practice, university education, continuous medical education and years of practice as experiences whereas prescribing attitude depends on age, sex and medical specialization. ${ }^{10}$ Lack of confidence, updating of knowledge on antibiotic resistance and negative self-refining attitude also contribute to wrong antibiotic prescribing behavior. ${ }^{13}$

Social opportunity (OSo): Medical education of Bangladesh was not adequately addressing the impending health issues. Negligible importance on antibiotics and AR issues in academic curriculum of undergraduate and postgraduate level causes poor antibiotic prescribing skills for future physicians. ${ }^{2}$ There is also a negligible emphasis on antibiotic use and resistance issues in different print $(0.02 \%-2.0 \%)$ and electronic media $(0-.6 \%)$ for disseminating awareness nationwide. ${ }^{2}$ The Early career of the prescribers follows the immediate influence of more-senior colleagues. However, the exercise of greater autonomy and personal experience cause major influence on antibiotic prescribing decisions with time. ${ }^{14}$ Even, peer and opinion leader perceptions and behaviors have a strong influence on prescribing behavior than local policy and guidelines. ${ }^{19}$ Healthcare systems are related to antibiotic misprescription behavior. It depends on the implementation status of antibiotic guidelines and quality of care by quality of providers in health care settings. ${ }^{10}$ In Bangladesh, hospital pharmacy, clinical pharmacy and community pharmacy practices are at nascent state. Usually, graduate pharmacists are engaged with pharmaceutical industry and diploma pharmacists enter into hospitals. Thus, there has an unmet need of skills of the staff in community and hospital pharmacies. Extreme lack of quality pharmacy professionals in health care settings leads to poor antibiotic use management. ${ }^{20}$ This leads to the clinical autonomy of doctors while decision-making on antibiotic prescription.

At the government level, the policymakers, lawmakers and regulators' reluctance on enacting a law to overcome inadequacy in rules and regulations to promote good prescribing practices lead to the irrational use of antibiotics in Bangladesh. Moreover, no relevant issues have been placed in 'National Health Policy 2010', 'National Drug Policy 2005', 'Drug (Control) Ordinance 1982' and 'Bengal Drug Act 1940' of Bangladesh. ${ }^{2}$ Health care policies (eg; 
Reimbursement), legal issues (eg; malpractice law) and regulatory practices (eg; OTC dispensing) are very important determinants of irrational antibiotic use behavior. ${ }^{10}$

Pharmaceutical companies (199 allopathic manufacturers) are the only organizations in Bangladesh to provide information of medicine to health personnel and produces $97 \%$ of total medicine in the local market. ${ }^{15}$ This information is often biased and inappropriate for promoting sales in the market. ${ }^{21}$ Pharmaceutical companies provide incentives on antibiotic prescription in contract with prescribers and even with organizations; hospitals and drug shops of Bangladesh, which are key determinants to antibiotic overuse. ${ }^{21}$ They are able to motivate prescribers with financial incentives through marketing strategies and policies. Moreover, there is no policy and regulation on prescribing antibiotics by informal practitioners and antibiotics selling without prescription although there are two organizations; Directorate General of Drug Administration (DGDA) and Pharmacy Council of Bangladesh (PCB) for regulating drugs and pharmacies in Bangladesh. ${ }^{15}$ Additionally, unqualified providers are rarely blamed for their poor practices owing to the fatalistic attitude of the villagers. The poverty influences the villagers to reach qualified professionals and incomplete antibiotic therapy. ${ }^{12}$

The common patient factors involved lack of knowledge between bacterial and viral infections, expectation, satisfaction and compliance with an antibiotic prescription, notions of antibiotics effectiveness, mutual recognition, patient's pressure, the possibility of losing patients are directly associated with prescribers antibiotic prescribing behavior. ${ }^{10,13,22}$ Consumer's ignorance, self-medication behavior, and OTC antibiotics selling aggravate misuse - 100, 000 doses of antibiotics had been dispensed without a prescription in one month at Rajbari district of Bangladesh. ${ }^{22}$

Besides, patient volume and limited time of prescribers both in

\section{Secondary analysis}

Table I Secondary analysis of determinants (summary)

\begin{tabular}{|c|c|}
\hline Capability & \\
\hline Physical & Psychological \\
\hline Unqualified prescribers & Misunderstanding of disease and antibiotic treatment \\
\hline Unqualified drug seller & Lack of confidence in diagnosis and no antibiotic prescribing \\
\hline \multirow[t]{2}{*}{ Ignorance of public } & Perceiving inability to risk/benefit \\
\hline & Knowledge gap and attitude \\
\hline \multicolumn{2}{|l|}{ Opportunity } \\
\hline Social & Physical \\
\hline $\begin{array}{l}\text { Weakness in health care policies; drug policy \& rules, legislation, and } \\
\text { regulation on antibiotic use }\end{array}$ & $\begin{array}{l}\text { Protocol and control systems for antibiotics prescribing and } \\
\text { use (antibiotic policy and guidelines) }\end{array}$ \\
\hline Socioeconomic status (poverty) & CPD training \\
\hline $\begin{array}{l}\text { Cultural beliefs (illness perception, family or friends belief, self-medication, } \\
\text { care seeking from informal providers) }\end{array}$ & Availability and accessibility of antibiotics \\
\hline AR pattern data and statistics & Diagnostic facilities \\
\hline Pharmacy practices & Incentives \\
\hline Drug promotion & Resources \\
\hline Antibiotic Information in society & Academic curriculum \\
\hline
\end{tabular}

public and private hospital settings are another major problem area. Average consultation time per patient in the primary care of Bangladesh is only 54 seconds. ${ }^{16}$ Few seconds' patients' description influence prescribers drawing general conclusions on antibiotic prescribing. Additionally, public service providers have less commitment because they are engaged with private sectors. ${ }^{20}$

Physical opportunity (OPh): Some organizational barriers to rational prescribing have been identified in health care settings similar to Bangladesh. They are the inadequacy of protocols and control systems; hospital formulary, evidenced-based antibiotic guidelines, limited permission to prescribe certain antibiotics, antibiotic order and stop order forms, antibiotic consultation. ${ }^{10,17}$ No or minimal facilities of microbial diagnosis, delayed culture sensitivity test results, and diagnostic uncertainty cause lack of confidence in outcome with narrow-spectrum antibiotics drive prescribers behavior to blind or empiric prescription. ${ }^{17}$

Automatic motivation (MA): Fear of losing patient and incentives from pharmaceutical companies promptly motivate prescribers to the antibiotic prescribing decision. ${ }^{17}$

Reflective motivation (MR): A matrix of societal, economic and health factors affect consumer's decisions. ${ }^{18} \mathrm{Up}$ to $80 \%$ of illness events are self-treated globally and antibiotic is very common. The beliefs of family or friends, the larger community beliefs, information from prescribers and dispensers, and promotional material contribute considerably to consumer's decision-making on antibiotic use. Some cultural and socioeconomic factors; living condition, vaccination coverage, social pressure are also linked with antibiotic use behavior. ${ }^{10}$ In Bangladesh, a significant number of doctors' have a low perception of patients condition (Chronic/ Acute and etiology) and fewer treatment beliefs (whether the antibiotics have necessity, effectiveness, efficacy, adverse effects, contraindications, toxic response)., ${ }^{2,3,12}$ 
Table Continued...

\section{Capability}

Clinical autonomy in decision-making

Peer opinion and patients pressure

Mutual recognition

Lack of education and training on rational prescribing

of antibiotics

Lack of antibiotic awareness

\section{Motivation}

\section{Automatic}

Fear of losing patients

\section{Pharmaceutical companies}

Availability of antibiotics in open market

\section{Reflective}

Perception of patients condition

Treatment beliefs

Personality and attitudes

Consumers beliefs

\section{Limitations}

The entire base of information is secondary data. Among major limitations, the limited number of published literature and scarcity of well-designed qualitative study on determinants influencing APB in Bangladesh were of concern. This review did not utilize any risk assessment tools to determine the risk of bias of the studies. Therefore, a generalization of findings on antibiotic prescribing behavioral determinants is tricky because of literature' sample size, study design, specific area of work within the country although having similar findings to the nearest country like India, Nepal, Pakistan and other LMICs. However, the scarcity of well-designed relevant qualitative studies in Bangladesh would enforce to design and conduct similar studies in immediate future for the development of pragmatic interventions to curb AR.

\section{Conclusion}

The authors concluded that there is a lack of awareness regarding antibiotic resistance, and appropriate antibiotic prescribing practices, which are prescribers' lack of able behavioral reflection. Additionally, diagnostic uncertainties, lack of provision of antibiotic guidelines and training, the absence of prescription monitoring systems, and patients' overcrowding are obstacles under social and physical opportunities to reduce the inappropriate antibiotic prescribing behavior. Furthermore, patients' ignorance on antibiotics and their expectation influence doctors' decision-making. Finally, deficiency of regulatory actions at social and policy level to plunge pharmaceutical's antibiotic promotion with misleading information and financial incentives, which significantly encouraged irrational antibiotics prescribing behavior from primary care to tertiary care. The determinants to inappropriate antibiotic prescribing should be controlled with pragmatic action plans adopted by stakeholders and policymakers to reduce the burden of antibiotic resistance in Bangladesh.

\section{Acknowledgements}

Authors are grateful to Leeds Institute of Health Sciences, University of Leeds, UK for supporting databases access for the literature search.

\section{Conflict of interest}

The author declares no conflict of interest.

\section{References}

1. Ahmed SM, Hossain MA, Chowdhury MR. Informal sector providers in Bangladesh: how equipped are they to provide rational health care? Health Policy Plan. 2009;24(6):467-478.

2. Rahman MS, Huda S. Antimicrobial resistance and related issues: An overview of Bangladesh situation. Bangladesh Journal of Pharmacology. 2014;9(2):218-224.

3. Khan MO, Chowdhury AK, Matin MA, et al. Effect of standard treatment guidelines with or without prescription audit on prescribing for acute respiratory tract infection (ARI) and diarrhea in some thana health complexes (THCs) of Bangladesh. Bangladesh Med Res Counc Bull. 2007;33(1):21-30.

4. Antimicrobial resistance: global report on surveillance. World Health Organization. 2014. 257 p.

5. Chin T. Health Policy Brief: Antibiotic Resistance. Health Affairs. 2015.

6. CDC's top ten: 5 health achievements in 2013 and 5 health threats in 2014. USA: CDC; 2014

7. Stålsby Lundborg C, Tamhankar AJ. Understanding and changing human behavior-antibiotic mainstreaming as an approach to facilitate modification of provider and consumer behaviour. Ups J Med Sci. 2014;119(2):125-133.

8. Tamhankar AJ, Nerkar SS, Patwardhan AP, et al. Antimicrobial resistance: from emerging threat to reality. India: Narosa Publishing House; 2009. p. 118-128.

9. Diwan V, Lundborg CS, Tamhankar AJ. Seasonal and temporal variation in release of antibiotics in hospital wastewater: estimation using continuous and grab sampling. PloS one. 2013;8(7):e68715.

10. Gould IM, van der Meer JW. Antibiotic policies: fighting resistance Springer; 2007.

11. Michie S, van Stralen MM, West R. The behavior change wheel: a new method for characterizing and designing behaviour change interventions. Implement Sci. 2011;6(1):42.

12. Gualano MR, Gili R, Scaioli G, et al. General population's knowledge and attitudes about antibiotics: a systematic review and meta-analysis. Pharmacoepidemiol Drug Saf. 2015;24(1):2-10.

13. Charani E, Castro-Sanchez E, Sevdalis N, et al. Understanding the determinants of antimicrobial prescribing within hospitals: the role of “prescribing etiquette". Clin Infect Dis. 2013;57(2):188-196. 
14. De Souza V, MacFarlane A, Murphy AW, et al. A qualitative study of factors influencing antimicrobial prescribing by non-consultant hospital doctors. J Antimicrob Chemother. 2006;58(4):840-843.

15. https://en.wikipedia.org/wiki/Pharmaceutical_industry_in_Bangladesh

16. Guyon AB, Barman A, Ahmed JU, et al. A baseline survey on use of drugs at the primary health care level in Bangladesh. Bull World Health Organ. 1994;72(2):265-271.

17. Faiz MA, Basher A. Antimicrobial resistance: Bangladesh experience. In Regional Health Forum. 2011;15(1):1-18

18. Fresle DA, Wolfheim C. Public education in rational drug use: a global survey. Geneva, Europe; 1997.
19. Schouten JA, Hulscher ME, Natsch S, et al. Barriers to optimal antibiotic use for community-acquired pneumonia at hospitals: a qualitative study. Qual Saf Health Care. 2007;16(2):143-149.

20. Alam GM, Shahjamal MM, Al-Amin AQ, et al. State of pharmacy education in Bangladesh. Tropical Journal of Pharmaceutical Research. 2013;12(6):1106-1112.

21. Islam MS. A review on the policy and practices of therapeutic drug uses in Bangladesh. Calicut Medical Journal. 2006;4(4):e2.

22. Roy J. Health status, treatment and drug use in rural Bangladesh: a case study of a village. Aust J Rural Health. 1997;5(2):70-75. 